\title{
Tadschikistan - ein Porträt
}

Als alle Völker der Erde von Gott ihr Land erhielten, waren die Tadschiken als Letzte an der Reihe. "Ich muß euch geben, was übriggeblieben ist, ich kann euch nur noch Berge bieten", sprach Gott. Das tadschikische Volk begann zu jammern: "Wie sollen wir in dieser Einöde nur leben?» Gott ließ sich durch die Klagenden erweichen und erwiderte: "Ich habe nur noch Berge, aber ich gebe euch ein Stück vom Paradies dazu.»

Tadschikische Sage

Mit dem Auseinanderbrechen der Sowjetunion sind bisher unbekannte zentralasiatische Republiken in den Brennpunkt des Weltinteresses gekommen. In einem strategisch heiklen Gebiet zwischen den Giganten Rußland und China, an der Nahtstelle des islamischen, europäischen und fernöstlichen Kulturraumes sowie in der Nähe des Bürgerkriegslandes Afghanistan, erlangten fünf Akteure - Kasachstan, Kirgisistan, Tadschikistan, Turkmenistan und Usbekistan - neue geopolitische Bedeutung. Alle Republiken erlebten eine Wiedergeburt des Nationalismus, der die während der russischen und sowjetischen Kolonialzeit hergestellten Bindungen zur Zentralregierung in Moskau bewußt zu kappen versuchte.

Im folgenden soll die Situation der Republik Tadschikistan erläutert werden, welche in vielen Belangen eine Sonderstellung einnimmt.

\section{Geographie}

Mit einer Fläche von $143100 \mathrm{~km}^{2}$ (FISCHER WELTALMANACH 1994) ist Tadschikistan die kleinste der einst sowjetischen Republiken Zentralasiens. Im Süden grenzt das Land an Afghanistan, im Osten an die chinesische Sinkiangregion und im Norden und Westen mit einer stark geschlungenen Grenzlinie an Kirgisistan und Usbekistan.

Über 90\% der Landesfläche Tadschikistans werden von Gebirgen eingenommen. Diese sind stark zertalt, schroff und zum Teil nur schwer zugänglich. Am ausgedehntesten ist der Pamir, welcher im Osten die halbe Landesfläche bedeckt. Hier liegen die höchsten Gipfel (Pik Kommunismus, $7495 \mathrm{~m}$ ü. M.) und längsten Gletscher (Lednik Fedschenko, $70 \mathrm{~km}$ ) der ehemaligen Sowjetunion. Im Ostpamir, an der Grenze zu China, liegt auf etwa $4500 \mathrm{~m}$ ü. M. eine Hochebene. Das Pamirgebiet bildet die Region Gorno Badachschan, welche eine gewisse politische Autonomie genießt.
Erschlossen wird diese Region durch den PamirHighway, welcher von der Hauptstadt Duschanbe an die afghanische Grenze, über Chorog, den Hauptort von Gorno Badachschan, bis nach Murgab im Ostpamir und schließlich ins kirgisische Osch führt. Das Wegstück zwischen Duschanbe und Chorog ist nur in den Sommermonaten passierbar.

Im Pamir und seinem westlichen Vorland entspringen die beiden Flüsse Vachsch und Pjandsch, welche später zum Amu Darja zusammenfließen und über weite Strekken die Südgrenze des Landes bilden. Der Amu Darja wendet sich auf usbekischem Gebiet gegen Nordwesten und mündet wie fast alle tadschikischen Flüsse in den Aralsee. Der zweite wichtige Zufluß des Aralsees, der Syr Darja, entspringt der Pamirnordabdachung auf kirgisischem Gebiet, durchquert anschließend das fruchtbare Ferganatal und legt bei Chodschand (früher Leninabad) eine kurze Strecke auf tadschikischem Gebiet zurück. Der zentrale Landesteil nördlich der Hauptstadt Duschanbe wird durch die von Osten nach Westen verlaufende, durch den Seravschan zweigeteilte Gissar-Turkestan-Kette geprägt. Ausläufer des Tienschan schließlich formen nördlich von Chodschand, der zweitgrößten Stadt des Landes, den Kuraminsker Rücken.

Südlich von Duschanbe verlaufen niedrige Hügelzüge, welche eine mächtige Lößbedeckung aufweisen. Sie trennen Duschanbe von den lokalen Zentren Kuljab und Kurgan Tjube im Süden des Landes. Im Südwesten münden die Täler in ein Tiefland, welches in die turkmenische Karakumwüste übergeht (ATLAS TADSCHIKSKOI SSR 1968).

Das Klima Tadschikistans ist aufgrund des starken Reliefs sehr unterschiedlich und nicht mit Durchschnittswerten zu charakterisieren. Die Täler mit ihren milden Wintern und trocken-heißen Sommern, in denen das Thermometer oft auf $+45^{\circ} \mathrm{C}$ steigt, können als subtropisch bezeichnet werden. In den unteren und mittleren Berglagen herrscht ein feucht-kühles Klima vor und in der Pamirregion ein Hochgebirgsklima, welches sich im Bereich der Ostpamirhochebene durch sehr tiefe Temperaturen (Mittel: $-2,6^{\circ} \mathrm{C}$, Min.: $-50^{\circ} \mathrm{C}$ ) und geringe Niederschläge $(68 \mathrm{~mm} / \mathrm{Jahr}$ ) auszeichnet (VON MAYDELL 1983).

Christian Hitz, dipl. Geograph, Limmatstr. 211, 8005 Zürich 


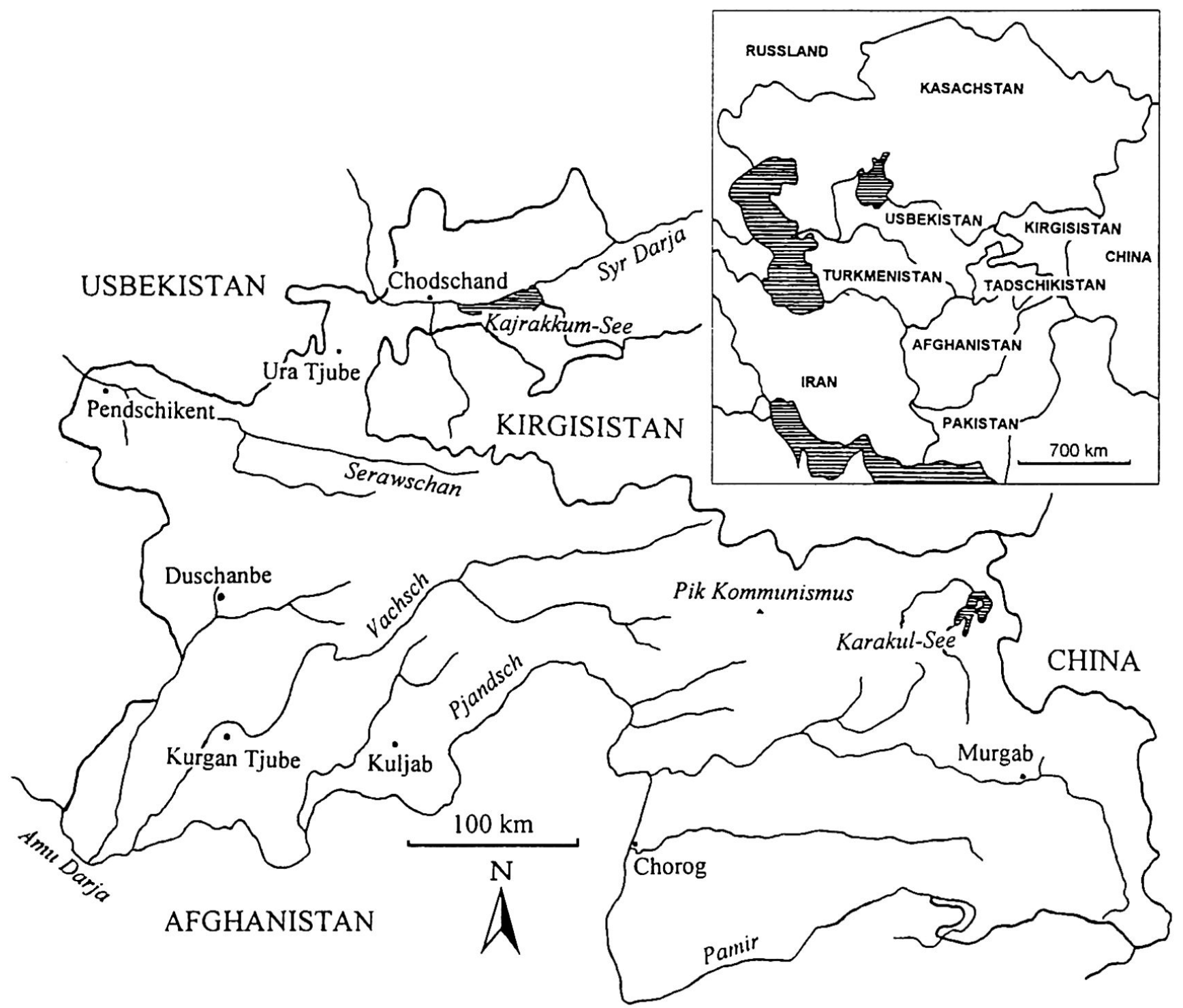

Abb.1 Übersichtskarte von Tadschikistan und Zentralasien.

Der gesamte zentralasiatische Gebirgsraum ist tektonisch noch keineswegs zur Ruhe gekommen. Mehrmals jährlich treten starke Erdbeben und Erdstöße auf (vON MAYDELL 1983). Diese große Erdbebengefahr führt dazu, daß in Duschanbe kaum Hochhäuser errichtet werden. Einstöckige Bauten sind in der Überzahl.

\section{Geschichte}

Das innerasiatische Zweistromland mit den Flüssen Oxus (Amu Darja) und Jaxartes (Syr Darja) war bereits im Paläolithikum besiedelt. Ungefähr eine Million Jahre alt sind die ältesten Spuren menschlicher Besiedlung (MUSEUM RIETBERG 1989).

Um 330 v. Chr. fiel Zentralasien in die Hände Alexanders des Großen, welcher in einem beispiellosen Siegeszug nach Osten vordrang. In Ura Tjube, einer Kleinstadt im Norden Tadschikistans, soll er geheiratet haben. Ruchschona, der Name seiner sagenumwobenen Braut, erfreut sich heute im Rahmen der nationalistischen Rückbesinnung großer Beliebtheit. Der Hellenismus hielt sich in Tadschikistan bis in die Mitte des 1. Jh. v. Chr. und wurde abgelöst durch eine Kultur der Nomaden, welche aus den weiten Steppen Mittelasiens kamen (MUSEUM RIETBERG 1989).

Seit dem 3. Jh. v. Chr. gehörten große Teile Zentralasiens zum persischen Sassanidenreich. Unter persischer Herrschaft erreichten Zentralasien und insbesondere die Städte Samarkand und Buchara einen kulturellen Höhepunkt. Mit dem zunehmenden Fernhandel gewannen die am Zugang zur Seidenstraße gelegenen Gebiete an Bedeutung. Aus jener Epoche stammt der Dichter Firdausi, der Autor des persischen Nationalepos (Königsbuch), dessen Statue heute an Lenins Stelle auf einem der Hauptplätze Duschanbes steht. Um 560 n. Chr. drangen 


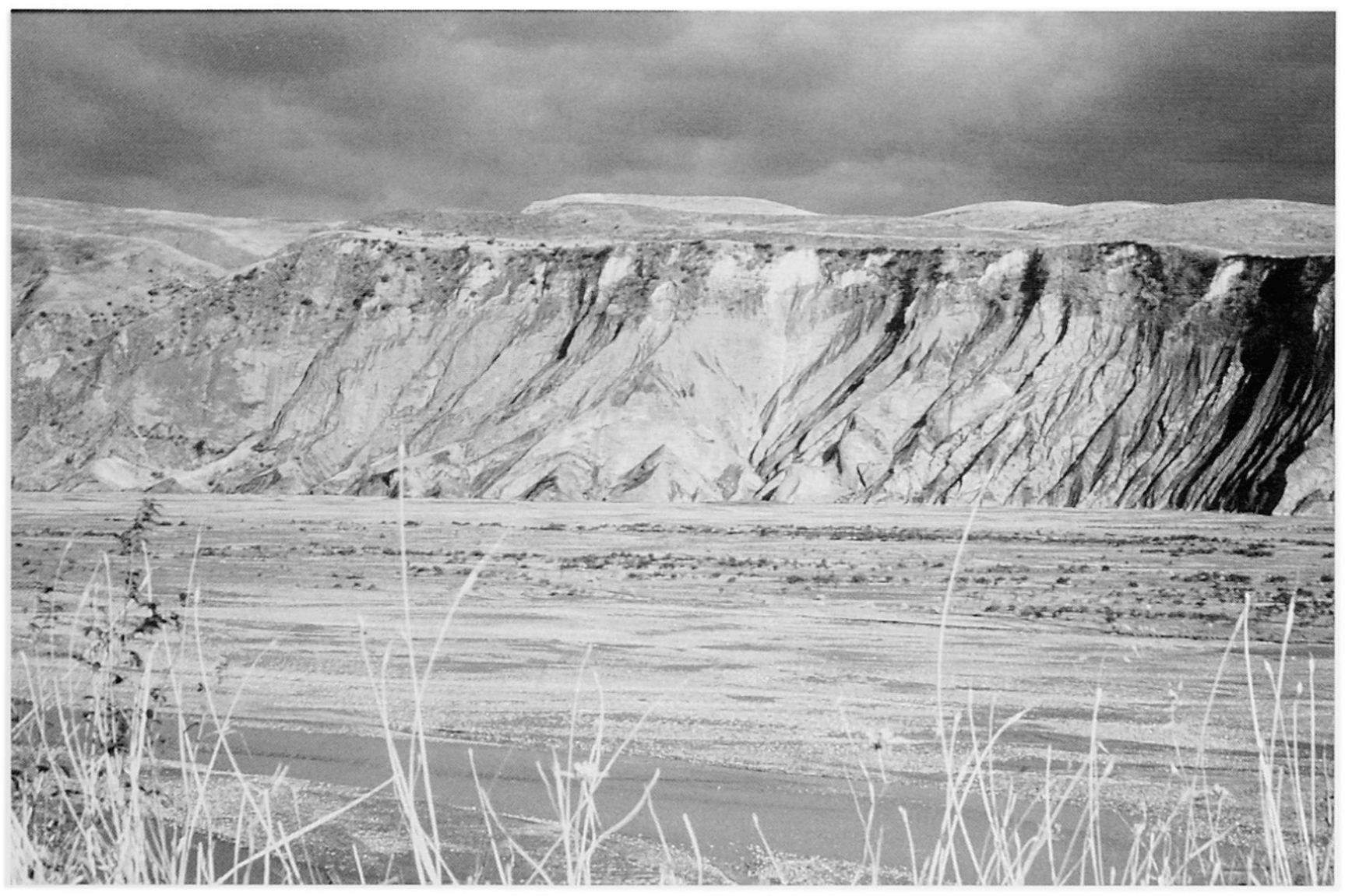

Abb. 2 Kysylsu - ein Fluß im Süden Tadschikistans (Aufnahme Christian Hitz).

die Türken in die innerasiatischen Steppenregionen ein. Zu Beginn des 8. Jh. n. Chr. stießen arabische Heere nach Zentralasien vor und verbreiteten im ganzen Gebiet eine neue, islamisch geprägte Kultur (MUSEUM RIETBERG 1989).

Anfang des 13. Jahrhunderts rissen die Mongolenheere Dschingis-Khans ganz Zentralasien in einen Strudel kriegerischer Ereignisse. Erst unter dem damals bekannten tadschikischen Herrscher Tamerlan kamen die Region und vor allem Samarkand als Hauptstadt seines Reiches zu neuem Glanz. Die beiden Städte Samarkand und Buchara, heute in Usbekistan liegend, aber überwiegend von tadschikischstämmiger Bevölkerung bewohnt, bildeten im späten Mittelalter wichtige weltliche und religiöse Zentren, welche in den gesamten arabischen Raum ausstrahlten. Aus dieser Blütezeit sind zahlreiche Moscheen und Medressen (Koranschulen) erhalten geblieben.

Die folgenden Jahrhunderte waren in Zentralasien durch wechselnde Einflüsse türkischer und persischer Herrschaft geprägt, bis nach dem Sieg Iwans des Schrecklichen in der Schlacht von Kasan (1552) die Bedeutung des Zarenreiches stark zunahm.

Die Region des heutigen Tadschikistan blieb aber ein Teil des Emirates von Buchara, bis schließlich 1870 russische Truppen an die Ufer des Amu Darja vordrangen. Nach der Russifizierung (Einführung des kyrillischen Alphabets) setzte die Sowjetisierung ein. 1924 wurde Tadschikistan der usbekischen Republik zugeordnet und das Bauerndorf Duschanbe (was soviel wie "Montagsmarkt» bedeutet) zur Provinzhauptstadt erklärt. 1929 erfolgte die Umwandlung Tadschikistans in eine eigenständige Republik. Bis in die vierziger Jahre dieses Jahrhunderts ist die Kollektivierung der Landwirtschaft vorangetrieben worden.

1991 erklärte sich Tadschikistan für unabhängig. Der heutige Staat hat sich mit Grenzen zu arrangieren, die von der Moskauer Zentralregierung in den zwanziger Jahren bei der Aufteilung des alten Turkestan willkürlich festgelegt wurden. Die Verwaltungseinheit von einst sieht sich als «Nationalstaat» in die Gegenwart katapultiert. Der Prozeß des fortschreitenden tadschikischen Nationalismus religiöser Prägung hatte zur Folge, daß sich im Lande seit Jahrzehnten vorhandene Spannungen zwischen verschiedenen Bevölkerungsgruppen entluden. Die Bewohner der Region Chodschand im Norden sowie jene aus dem Gebiet von Kuljab im Süden sahen ihre zu Sowjetzeiten gefestigte Machtbasis in der Regierung gefährdet (NZZ 13.8.1993).

Der 1992 ausbrechende Bürgerkrieg kann somit auch nicht auf das oft angeführte Schema "Altkommunisten gegen islamische Fundamentalisten» reduziert werden. 
Er läßt sich eher auf verworrene Clanstreitigkeiten, regionale wirtschaftliche Interessen und unter dem sowjetischen Regime unterdrückte Ansprüche einzelner Talschaften zurückführen. Nachdem der Krieg über 20000 Todesopfer gefordert, etwa 600000 Menschen in die Flucht getrieben hat (NZZ 5.5.1993) und Folter und barbarische Morde zur Tagesordnung werden ließ, beruhigte sich die Lage 1993 ein wenig, und der Krieg wurde für beendet erklärt. Damit waren aber die Ursachen der Auseinandersetzung noch lange nicht beseitigt. Die fundamentalistische Opposition wurde militärisch besiegt, zog sich auf afghanisches Territorium zurück und bildete dort eine Exilregierung. Bis heute wird immer wieder von Scharmützeln aus der Grenzregion berichtet, doch die Kriegsintensität mit fünf bis zehn Todesopfern pro Woche scheint zu gering, um internationale Aufmerksamkeit auf die tadschikische Tragödie zu lenken.

Am 6. November 1994 wurden Präsidentschaftswahlen durchgeführt. Die Vereinten Nationen, welche ein Büro in Duschanbe unterhalten, distanzierten sich schon vor den Wahlen von dem Urnengang, da schon die Wahlvorbereitungen keineswegs frei und fair verliefen (NZZ 8.11.1994). Der neu gewählte Präsident heißt Imamali Rachmanow. Der große Einfluß der Herkunftsregion der Kandidaten auf den Ausgang der Wahl läßt sich aus den Resultaten ableiten: Während Rachmanow in seiner Heimatstadt Kuljab im Süden des Landes 99\% der Stimmen erhalten haben soll, vereinte sein Gegner Abdulmalek Abdulladschanow in der Region Gorno Badachschan $96 \%$ der Stimmen auf sich. Nach den Wahlen war im russischen Fernsehen von Wahlbetrug die Rede. Die Situation in Duschanbe blieb überraschend ruhig. Dies, nachdem in den Tagen vor den Wahlen mehrere Bombenanschläge in der Hauptstadt ein Klima der Unsicherheit geschaffen hatten (TA 5.11.1994).

Am 26. Februar 1995 wurde das neue Parlament gewählt. Aufgrund des im Dezember verabschiedeten Wahlgesetzes waren außer den Regierungsparteien alle politischen Gruppierungen praktisch von der Wahlteilnahme ausgeschlossen. Auch der ehemalige Präsidentschaftskandidat Abdulladschanow wurde von der Wahlkommission nicht zur Wahl zugelassen (TA 26.2.1995). Heute wird die große Mehrheit des Parlaments durch die neokommunistischen Anhänger des Staatschefs gebildet, welche vorwiegend aus Kuljab stammen.

Bis heute bleiben 25000 russische Soldaten an der afghanischen Grenze stationiert. Deren Anwesenheit wird durch die russische Regierung mit der Notwendigkeit des Schutzes der russischen Bevölkerung in Tadschikistan gerechtfertigt (NZZ 15.7.93). Weiterhin setzen beide Konfliktparteien auf Konfrontation. Militärausgaben, welche nach offiziellen Angaben $60 \%$ des tadschikischen Staatshaushaltes verschlingen, und die erneute Unterordnung unter die Fuchtel Moskaus sind der Preis, den Duschanbe bezahlt, um zusammen mit russischen Truppen die Rebellen im Süden in Schach zu halten. Auch wenn sich die politische Lage in der letzten Zeit etwas beruhigt hat, scheint eine stabile Friedenssituation in weiter Ferne zu liegen.

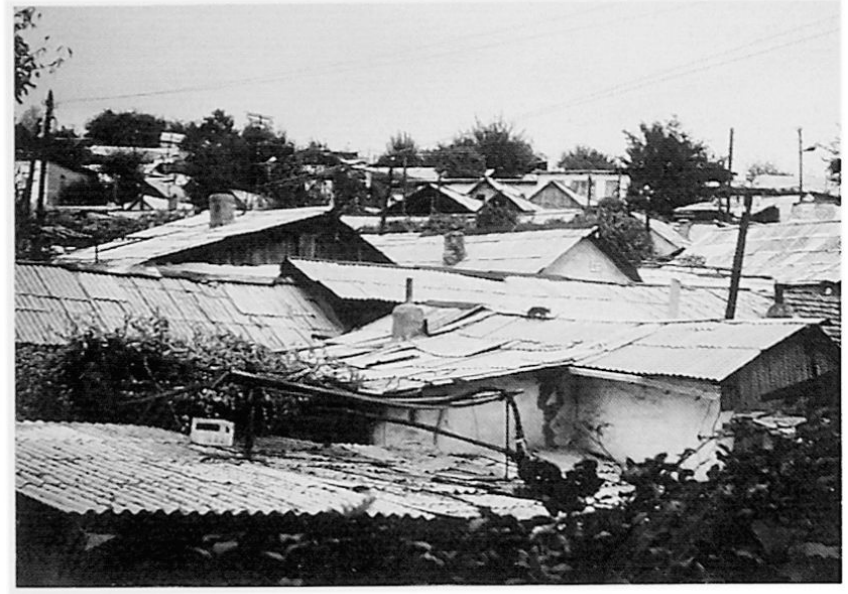

Abb. 3 Dächer in einem Außenbezirk von Duschanbe. Bedingt durch die hohe Erdbebengefahr wird meist nur einstöckig gebaut (Aufnahme Christian Hitz).

\section{Bevölkerung}

Die Bevölkerung des Landes setzt sich aus 62\% Tadschiken, 23\% Usbeken, 8\% Russen, 1,4\% Tataren und 1,3\% Kirgisen zusammen und zählt 5,6 Millionen Menschen (FISCHER WELTALMANACH 1994). Diese starken ethnischen Minderheiten finden sich in den meisten ehemals sowjetischen Zentralasienrepubliken. Sie lassen sich auf die willkürlichen Grenzziehungen zurückführen, welche ohne Rücksichtnahme auf die historische Entwicklung gezeichnet wurden. Weitere starke Minderheiten wie Koreaner, Juden und Deutsche resultieren aus Umsiedlungsaktionen der Stalinära.

Der Anteil der Bevölkerung Tadschikistans an der Gesamtbevölkerung der Sowjetunion nahm von 1940 bis 1987 von $0,8 \%$ auf $1,7 \%$ zu. Das Wachstum liegt somit wesentlich über dem Durchschnitt der ehemaligen Sowjetunion. Etwa zwei Drittel der Bevölkerung leben im ländlichen Gebiet (BATER 1989). Bemühungen um eine stärkere Urbanisierung waren bisher nur bedingt erfolgreich. Die Bevölkerung ist innerhalb der Republik sehr ungleichmäßig verteilt. Drei Viertel aller Menschen leben in den Tallagen bis $1000 \mathrm{~m}$ ü. M., vor allem im Süden des Landes, um Duschanbe und in der Region Chodschand. Das Pamirgebiet ist mit einer Bevölkerungsdichte von unter einer Person pro Quadratkilometer praktisch menschenleer (VON MAYDELL 1983).

Im Gegensatz zu allen anderen ehemaligen Sowjetrepubliken, in denen Turksprachen vorherrschen, sprechen die Tadschiken einen persischen Dialekt. Russisch hat sich als Geschäftssprache durchgesetzt und wird von über einem Viertel der Bevölkerung fließend beherrscht (BATER 1989). Gemeinsam ist all diesen Ländern die sunnitische Ausprägung des Islam. Die etwa 200000 kirgisischstämmigen Bewohnerinnen und Bewohner der Region Gorno Badachschan, die Pamiri, sind der ismaelitischen Glaubensgemeinschaft zuzurechnen. 


\section{Wirtschaft}

Die heutige tadschikische Wirtschaftsstruktur muß als Ergebnis sowjetischer Planwirtschaft gesehen werden. Im Rahmen der gesamtsowjetischen Volkswirtschaft hatten die zentralasiatischen Republiken die Rolle der Rohstoffproduzenten inne. Die Weiterverarbeitung erfolgte praktisch ausschließlich im Norden der Sowjetunion. Steigende Weltmarktpreise für Baumwolle veranlaßten das staatliche Planungsministerium Gosplan dazu, den Schwerpunkt der agrarischen Produktion Mittelasiens auf Baumwollplantagen zu legen (BADENKOV 1994). Die ökologischen Konsequenzen des zur Erreichung der ehrgeizigen Produktionsziele stark geförderten Bewässerungsanbaus sind hinreichend bekannt.

Die engen Verbindungen zwischen den Sowjetrepubliken zeigten sich auch im Bereich der Schwerindustrie: Aufgrund der hohen Elektrizitätsproduktion durch die Wasserkraftnutzung wurde in Tadschikistan die energieintensive Aluminiumproduktion angesiedelt. Roh- und Endprodukte wurden importiert respektive exportiert. Die inhomogene Wirtschaftsstruktur mit einer kleinen Anzahl von Großbetrieben dominiert durch die forcierte Kollektivierung teilweise auch die landwirtschaftliche Produktion. Trotz starker Mechanisierung arbeiten noch heute $40 \%$ der Bevölkerung im primären Sektor, der vor allem Baumwolle, Seide und Fleisch produziert (LEwIS 1992).

Der Niedergang der Wirtschaft ist die Kehrseite der politischen Unabhängigkeit. Als äußerst schwierig erweist sich die Abkoppelung von der zerfallenden Sowjetunion. Tadschikistan, schon zu Sowjetzeiten eine der ärmsten Republiken, erlebte einen dramatischen Einbruch der Produktion: die Industrieproduktion sackte in den letzten Jahren auf die Hälfte des Volumens ein (NZZ 18./ 19.3.1995). Von diesem Rückgang ist auch die landwirtschaftliche Produktion betroffen, welche 1992 um 45\% schrumpfte. Gleichzeitig stiegen in diesem Jahr die Konsumentenpreise um 910\% (NZZ 16.6.1993). Plötzlich durchtrennte Zulieferungsströme lassen ganze Produktionszweige stillstehen. So mußte die Schmuckfabrik von Duschanbe bereits $40 \%$ der Angestellten entlassen, weil aufgrund der Liquiditätskrise des Landes kaum mehr Importe getätigt werden können. Es mangelt an Bleiglassteinen aus der ehemaligen Tschechoslowakei, an russischem Kupfer und an Gas, welches für die Lötmaschinen benötigt wird. Etwas resigniert sieht der Fabrikdirektor die Zukunft des Unternehmens in der Nudelproduktion.

Verstärkt wird die Krise der tadschikischen Wirtschaft durch den Exodus von fast 400000 europäischen ExSowjetbürgern, die mehrheitlich als Fach- und Verwaltungsangestellte tätig waren (NZZ 13.8.1993).

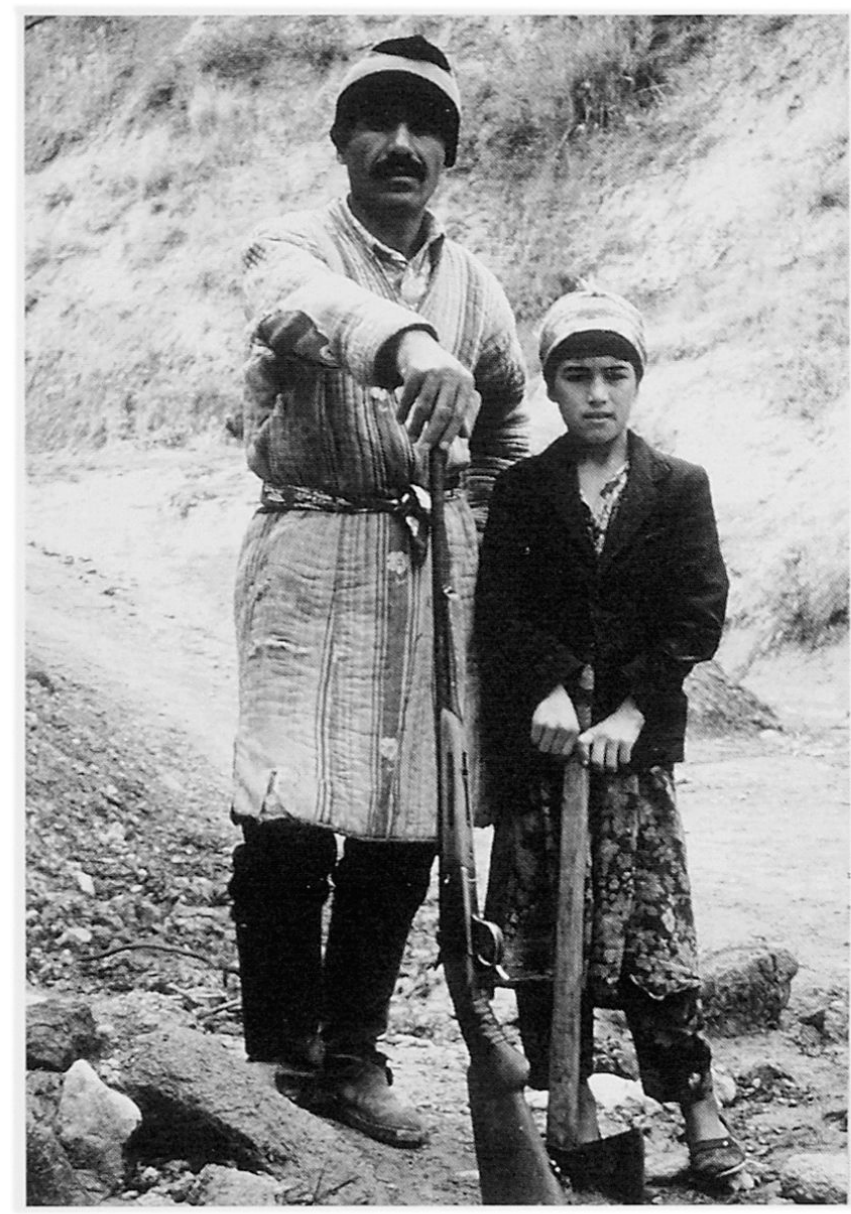

Abb. 4 Jäger mit seiner Tochter. Er ist auf dem Weg in die Berge, um wilde Hühner zu jagen (Aufnahme Christian Hitz).

\section{Alltagsleben}

Die Verkehrssituation befindet sich im ganzen Land in einem desolaten Zustand. Bedingt durch den Treibstoffmangel zirkulieren die öffentlichen Busse in Duschanbe nur noch sporadisch. Erscheint doch einmal ein Fahrzeug, läßt sich oft nur noch eine Mitfahrgelegenheit ergattern, indem man sich draußen an den Türrahmen hängt. Der Treibstoffmangel wirkt sich auch auf die Müllentsorgung aus. An den Straßenrändern türmen sich Abfallhaufen, nehmen von Tag zu Tag mehr Platz ein und breiten sich bis in die Straßen aus. Bei der Versorgung mit Grundprodukten ergeben sich immer öfter Engpässe. Die Warteschlangen vor den billigen staatlichen Brotgeschäften bilden sich schon um Mitternacht und werden, um Unruhen zu verhindern, von Sicherheitskräften bewacht.

Gemäß offiziellen Angaben soll die Arbeitslosigkeit 20\% betragen (FISCHER WELTALMANACH 1994). Die tatsächlichen Zahlen dürften wesentlich höher liegen. Zudem sind viele Betriebe nicht mehr in der Lage, den Arbeitnehmern ihre Löhne auszuzahlen. Die Entrichtung von Salären an Staatsangestellte ist angeblich seit Februar 1994 eingestellt. 


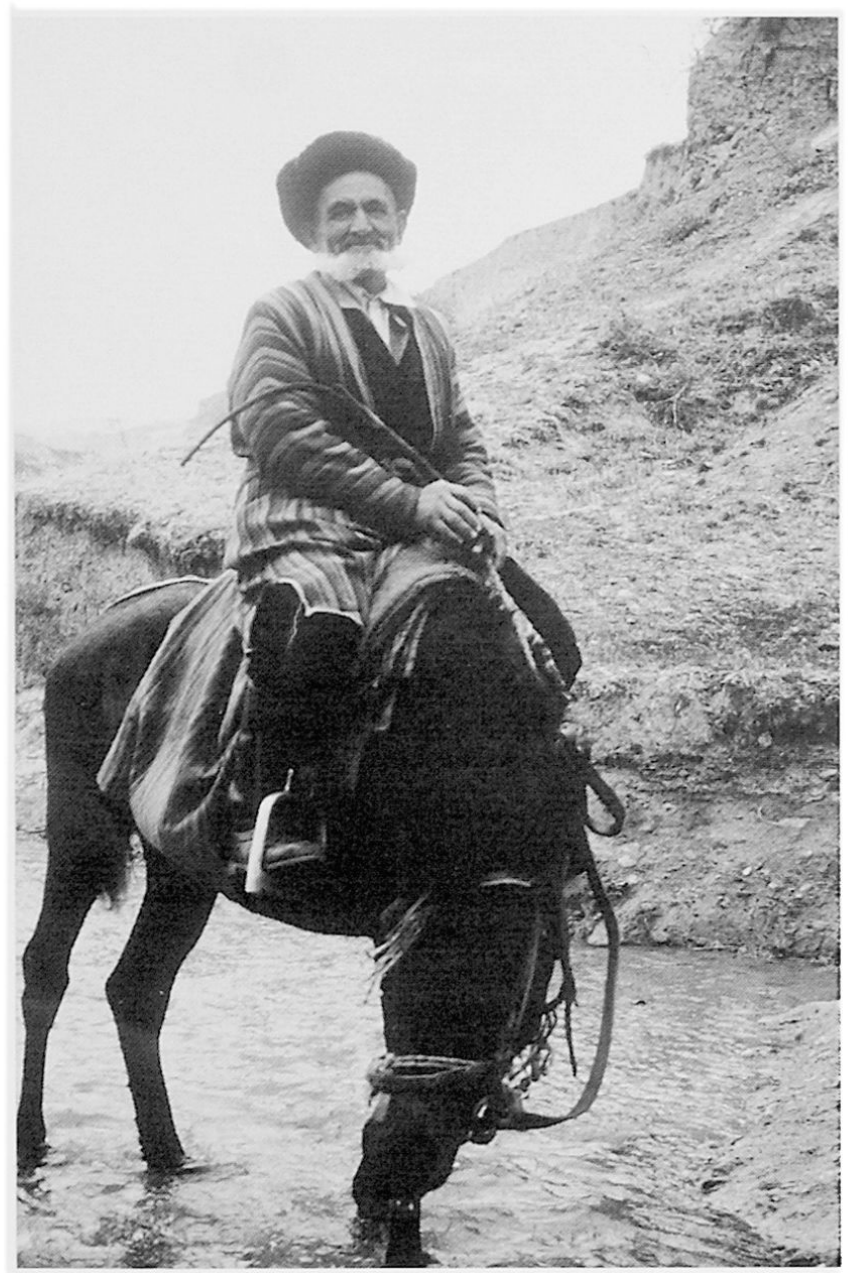

Abb. 5 Reiter in traditionellem Mantel, welcher Tschapán genannt wird. In den tadschikischen Bergen ist das Pferd ein oft benutztes Fortbewegungsmittel (Aufnahme Christian Hitz).

Schon 1991 lebten 87\% der Bevölkerung unter der Armutsgrenze (FISCHER WELTALMANACH 1994). Es gibt keinen Grund zur Annahme, daß dieser Anteil seither abgenommen hat.

Politische Instabilität und wachsende Armut sind mit verantwortlich für die zunehmende Kriminalität und Korruption, welche zusehends auch das Alltagsleben tangieren. Nach den Bürgerkriegsjahren ist es schon zur Selbstverständlichkeit geworden, daß die Öffentlichkeit nach Einbruch der Dunkelheit gemieden wird. Die einst pulsierenden Straßen Duschanbes sind menschenleer, die traditionellen Teestuben, Tschaichanas genannt, sind geschlossen. Mit stoischer Ruhe wird hingenommen, daß an den häufig anzutreffenden Straßensperren der Armee immer wieder einige Liter Benzin oder ein paar tausend Rubel abgegeben werden müssen.

Rückhalt in der Krise bietet der einheimischen Bevölkerung der enge Zusammenhalt der traditionellen Großfamilie, welche sich um das Wohlergehen sämtlicher Mitglieder kümmert. Mit einer Vielzahl von kleinen Ge- schäften und Tätigkeiten versucht man sich den Lebensunterhalt zu verdienen: in Kellern wird illegal Wodka gebrannt, oder Studierende verpacken offen gekaufte Zigaretten in gesammelte Schachteln von Westmarken.

Wesentlich schlechter sieht die Situation für Teile der russischen Bevölkerung aus. In einem Land, welches nie zu ihrer Heimat geworden ist und dessen Sprache sie nie zu lernen brauchten, fehlt ihnen das nötige Beziehungsgefüge, um die kleinen Probleme des Alltags zu lösen.

Die sich ständig verschlechternde Wirtschaftssituation bildet den Nährboden für eine rückwärtsgerichtete Entwicklung. Viele Menschen in Tadschikistan sehnen sich heute nach der alten Sowjetunion, in der eine klare Ordnung und gute wirtschaftliche Verhältnisse herrschten. Vielen macht der Abstieg von einer Weltmacht zu einem der ärmeren Länder zu schaffen. So ist es auch nicht erstaunlich, daß die Insignien der kommunistischen Ära in Tadschikistan Bestand haben. Am Ufer des Syr Darja, in der Stadt Chodschand, steht eine 30 Meter hohe Leninstatue. Mit erhobenem Arm und energischem Blick zeigt er seinem Volk den Weg in die Zukunft. Kaum jemand denkt daran, dieses Relikt zu entfernen.

Tadschikistan geht wie die anderen ehemals sowjetischen Zentralasienrepubliken einer unsicheren Zukunft entgegen. Mit denkbar schlechten Voraussetzungen versuchen diese Länder, den Anschluß an den Weltmarkt zu schaffen. Trotz seines Namens liegt Zentralasien nicht im Mittelpunkt des Weltgeschehens. Die im Pamir entspringenden Flüsse strömen nicht zu einem Welthafen, sondern versickern in den weiten Steppen.

\section{Literatur}

ATLASTADSCHIKSKOISSR(1968): Atlas der sozialistischen Sowjetrepublik Tadschikistan, Moskau-Duschanbe.

BADENKOV, J., GUNJA, A., LINDER, P. (1994): Traditionelle Wirtschaftsweise und Strukturwandel in einem peripheren Gebirgsraum am Beispiel Jagnob/Tadschikistan. In: Mitteilungen der fränkischen geographischen Gesellschaft, Band 41, Erlangen.

BATER, H. J. (1989): The Sovjet Scene: A Geographical Perspective. London.

BOZDAG, A. (1992): Das Erbe der UdSSR: Islam und Politik in Zentralasien. In: Jahrbuch Dritte Welt 1993, Beck'sche Reihe 486, München.

FISCHER WELTALMANACH (1994): Frankfurt a. M.

LEWIS, R. A. (1992): Geographic Perspectives on Sovjet Central Asia. London.

MUSEUM RIETBERG (1989): 2000 Jahre Kunst am OxusFluß in Mittelasien. Neue Funde aus der Sowjetrepublik Tadschikistan. Zürich.

NZZ: Neue Zürcher Zeitung: diverse Ausgaben.

TA: Tages-Anzeiger: diverse Ausgaben.

VON MAYDELL, H.-J. (1993): Mitteilungen der Bundesforschungsanstalt für Forst und Holzwirtschaft, Hamburg: Forstund Holzwirtschaft der Sowjetunion, Teil 4. Kasachstan und die Mittelasiatischen Sowjetrepubliken. 